\section{Scoring endoscopic disease activity in IBD: artificial intelligence sees more and better than we do}

With great interest we read the review article of Lacucci et al on advanced endoscopic techniques in IBD. ${ }^{1}$ The authors clearly recognise the current limitations of the different endoscopic scoring tools for disease activity in IBD and provide potential solutions with new advanced techniques. We fully agree that interobserver variability is problematic for treatment planning and IBD drug development and we lack objective definitions of endoscopic remission that predict further disease course in both Crohn's diseaseand ulcerative colitis (UC). Scoring systems were mostly developed at the end of the previous century, based on evaluations with fiberopticendoscopes or poor quality white light video-endoscopes. Intrinsically, it could be anticipated that by seeing more details and nuances such as with virtual chromo-endoscopy, this could result in a superior assessment of the disease activity in IBD. The data provided in the review by Lacucci et al indeed support this assumption in strict clinical trial setting. But one important thing has not been taken into account: the subjectivity of the human being. To give an example: when using a simple scoring system like the four scale Mayo endoscopic sub-score low interobserver agreement has been observed. ${ }^{23}$ Training programmes or standardised scoring conventions can improve this but will never overcome the disagreement. ${ }^{45}$ The Picasso score is an elegant example of a new UC activity score that optimally exploits the potential of the advanced imaging techniques. ${ }^{6}$ It discriminates subtle endoscopic features like crypt architecture and (sub)-mucosal vascular structures. This detailed judgement leads to good correlation with histological disease activity. ${ }^{6}$ But the performance of the Picasso in 'real life' is inferior to that during the development phase of the score. ${ }^{7}$ Moreover, no data are available on the use of these advanced endoscopic scores in real world practice where the endoscopist is even more biased by the clinical assessment of the patient. So, as long as we still rely on the human brain for the interpretation of the endoscopic image, we will never overcome the old hurdles of subjectivity leading to interobserver variability. For this, and without being too pessimistic, we believe that the applicability of advanced endoscopic techniques in the evaluation of disease activity in IBD has not such a bright future. To achieve a real paradigm shift one needs to go beyond the human being. Excluding the human brain will provide an objective operator-independent score that can be used by every clinician without specific training. There are two potential ways of doing this. First, computer learning algorithms can be applied to accurately interpret endoscopic images. Pilot trials on the use of a convolutional neural network have shown promising results. ${ }^{89}$ This type of computer aided diagnosis performs well in the evaluation of the Mayo endoscopic sub-score, but the problem is that everything depends on the data that trained the algorithm (ie, the Mayo endoscopic sub-score). Neural networks should ideally be trained by the most objective score (eventually based on advanced imaging techniques) provided through a central reading consensus. The second option might be superior. We performed image analysis by extracting pixel data from high definition images and integrated pattern recognition in the algorithm. These unbiased image data can be linked further to histological data. By doing so, we recently demonstrated that this approach leads to strong correlation with histology, which is known to be the best predictor of sustained clinical remission in UC. ${ }^{10}$ To avoid disappointment in the utopic quest for objective endoscopic scoring systems, let us ask the computers to do the work. Computers are more consistent and patientin image interpretation than the human eye and brain.

\section{Peter Bossuyt, ${ }^{1,2}$ Séverine Vermeire, ${ }^{1}$ Raf Bisschops ${ }^{1}$}

${ }^{1}$ Department of Gastroenterolgy and Hepatology, Universitaire Ziekenhuizen Leuven, Leuven, Belgium ${ }^{2}$ Departement of Gastroenterology, Imelda Hospital, Bonheiden, Belgium

Correspondence to Dr Peter Bossuyt, Department of Gastroenterolgy and Hepatology, Universitaire Ziekenhuizen Leuven, Leuven, Belgium; peter.bossuyt@imelda.be

Contributors PB: wrote the manuscript and did the literature review.SV:critically reviewed the manuscript. $r B$ : critically reviewed the manuscript and supervised the study.

To cite Bossuyt P, Vermeire S, Bisschops r. Gut epub ahead of print: [please include Day Month Year]. doi:10.1136/gutjnl-2019-318235

Received 7 January 2019

revised 22 March 2019

Accepted 26 March 2019

\section{RefeRences}

1 Lacucci M, Furfaro F, Matsumotot,etal.Advanced endoscopic techniques in the assessment of inflammatory bowel disease: new technology, new era. Gut 2018. doi: 10.1136/gutjnl-2017-315235. [epub ahead of print].

2 Fernandes Sr, Pinto J, Marques da Costa P, et al. Disagreementamong gastroenterologists using the mayo and rutgeerts endoscopic scores. Inflamm Bowel Dis 2018;24:254-60.

3 Daperno M, Comberlato M, Bossa F, et al. Interobserver agreement inendoscopic scoring systems: preliminary report of an ongoing study from the Italian Group for Inflammatory Bowel Disease (IG-IBD). Dig Liver Dis 2014;46:969-73.

4 Daperno M, Comberlato M, Bossa F, etal.training Programsonendoscopic Scoring Systemsfor Inflammatory Bowel Disease Lead to a Significant Increase in Interobserver Agreement Among Community Gastroenterologists. J Crohns Colitis 2017;11:556-61.

5 Dubcencoe, Zou G, Stitt L, et al. effect of standardised scoring conventions on inter-rater reliability in the endoscopic evaluation of Crohn's disease. J Crohns Colitis 2016;10:1006-14.

6 Lacucci M, Daperno M, Lazarev M, et al. Development and reliability of the new endoscopic virtual chromoendoscopy score: the PICaSSO (Paddington International Virtual ChromoendoScopy ScOre) in ulcerative colitis. Gastrointest Endosc 2017;86:1118-27.

7 Lacucci M, Lazarev M, tontini G, et al. Sa1983 - a prospective multicenter "real Life" International validation study of the picasso endoscopy scoring system againist histologic scoring system to define mucosal healing in ulcerative colitis. Gastroenterology 2018;154:S-436-0.

8 Ozawat, IshiharaS, Fujishiro M, et al. Novel computerassisted diagnosis system for endoscopic disease activity in patients with ulcerative colitis. Gastrointest Endosc 2019;89.

9 Zezos P, Borowski K, Bajaj G, et al. 439 - toward computer-based automated mayo score classification in ulcerative colitis through classical and deep machine learning. Gastroenterology 2018;154:90-9.

10 Bossuyt P, Nakase H, Vermeire S, et al. 436 Automated digital calculation of endoscopic inflammation in ulcerative colitis: results of the red density study. Gastroenterology 2018;154:S90-8. 